\title{
An Approach for the Financial Viability Assessment of Cloud Migration Projects
}

\author{
Kent Ramchand \\ Swinburne University of Technology \\ kramchand@swin.edu.au
}

\author{
Mohan Baruwal Chhetri \\ CSIRO's Data61, and
}

Swinburne University of Technology

mohan.baruwalchhetri@data61.csiro.au

\author{
Ryszard Kowalczyk \\ Swinburne University of Technology, and \\ Systems Research Institute \\ Polish Academy of Sciences \\ rkowalczyk@swin.edu.au
}

\begin{abstract}
Organisations moving legacy applications to the public cloud are increasingly having to reassess their decisions post-migration due to budget overruns resulting from insufficient planning and limited understanding of the constraints with the public cloud operating model. To date, there has been limited research on the assessment of public cloud suitability for legacy applications, which can lead to incomplete analysis and inappropriate platform choices and high-risk cost estimates. To address these shortcomings, we extend our previously proposed Cloud Decision Framework by incorporating a generalised Financial Viability Assessment process and methodology to help decision-makers make more efficient and effective migration decisions. The framework includes an automated approach for financial viability assessment using predefined application sizes via a Parametric Estimation Model. The proposed approach is validated by application to two real-world, anonymised case studies with results indicating that it is more likely to reduce budget overruns and increase productivity compared to current approaches.
\end{abstract}

\section{Introduction}

Market research indicates that the uptake of cloud computing is on the rise; according to Flexera [1], twenty percent of global enterprises invested more than $\$ 20 \mathrm{M}$ on public cloud usage in 2019. Whilst there are benefits in the adoption of cloud technologies [1], the migration to public cloud without a pragmatic approach and use of an appropriate framework can lead to unanticipated cost overruns or missed opportunity costs due to the delayed delivery of migration projects [1] [2]. Post-migration bill shock is a common symptom identified in Accenture's Cloud Survey of 200 IT Executives [3], where 65 percent of respondents had not achieved the anticipated cost savings from cloud adoption. This savings gap can be driven by several hidden costs not anticipated in the planning phase, including challenges with the compatibility of the application to a public cloud platform, and high performance storage requirements of the application that cannot be met by public cloud.

A recent literature study [4] analysing 53 articles identified that there is no consistent body of research for cloud economics and a clear research gap regarding cloud computing costs. Similarly Gartner's findings were that a new cloud operating model imposes new requirements, which incur additional costs that must be accounted for but are currently overlooked when building a business case for cloud migration [5]. To address this gap an appropriate framework and methodology is needed in the planning phase to (a) understand the application architecture and characteristics that determine the selection between public or on-premise private clouds, and (b) capture the platform associated hidden costs [3] [4] [5].

The success of cloud migration projects depends upon efficient and coordinated decision-making as part of a broader IT governance structure [6]. The recommended time spent on planning for a cloud migration project should be as high as $90 \%$ of the total effort [7] compared with $30 \%$ for traditional projects [8]. As shown in Fig. 1] (stage d), a critical step in the planning phase is the Rough Order of Magnitude (ROM) estimation [9] of cloud costs that covers any or all service models (i.e., IaaS, PaaS or SaaS) according to the enterprise's application migration plan. A ROM estimation is used because a detailed examination of each application's component migration requirements is unlikely to have occurred at this early stage. When conducting estimation, an enterprise depends upon the online cloud service provider calculators [10] [11] [12] [13] and current Cloud Computing Total Cost of Ownership (TCO) models proposed by researchers [14] [15]. However, not all costs are captured when compared with the actual costs charged by a public/on-premise-private cloud provider or incurred by the enterprise. For 


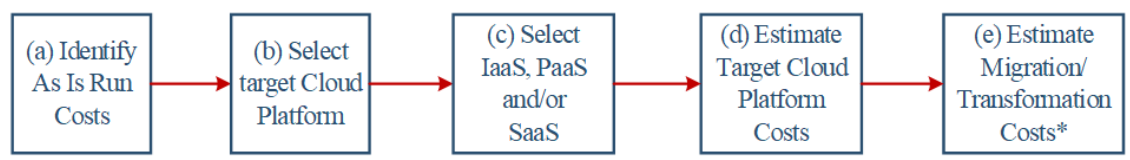

Figure 1. Planning stages of application migration to the cloud.

example, existing cloud service provider calculators do not consider the replacement of legacy development tools that are not suitable or supported in a cloud native environment, nor the required training costs of staff.

This paper extends our previously proposed Cloud Decision Framework (CDF) [16] [17] [18], by incorporating a generalised financial viability assessment (FVA) process and methodology to assist key decision-makers make more efficient and effective cloud migration decisions. The FVA explicitly captures both the service provider cost items and hidden cost items. The service provider costs used in the FVA calculation are taken from the market leaders in public and private cloud, including Amazon Web Services (AWS) and Microsoft Azure, which own 51\% and $13.3 \%$ of the market share respectively [19], and DELL, which owns $48 \%$ of the on-premise private cloud market share [12].

The FVA methodology captures the IaaS, PaaS and SaaS costs, after applying any associated enterprise agreement discounts for public cloud. Each cloud service model has a different commercial model; IaaS charges are based on resources provisioned, PaaS charges are based on service consumption, and SaaS charges are via subscription. For private cloud, costs are typically amortised over a 5-year period because enterprises are more likely to opt for the 5-year option since a hardware refresh is usually not required within that period and the monthly payment is lower when costs are amortised over a longer period.

The FVA is supported by a Parametric Estimation Model (PEM), which contains the estimates for cloud costs based upon application sizing. PEMs support the automated generation of cost forecasts based on pre-determined cloud resources and hidden costs such as professional services, software licenses, network connectivity, backup, management and monitoring. The reason for using a PEM based approach is to encourage sharing and reuse of PEMs developed by experts, for the breadth of applications that can be migrated to the cloud, by the wider community via an open repository.

The main contributions of this paper are as follows:

1. A cloud migration and financial viability assessment framework that enables a cloud adopter to (a) select the characteristics that are important for an application being targeted for Cloud Computing, (b) generate a cloud platform recommendation based on the selected characteristics, and (c) perform a FVA of the recommended platform. This enables cloud adopters to iteratively refine the characteristics for their platform and arrive at an affordable option that did not present itself initially.

2. A Parametric Estimation Model (PEM) for application sizing to support automated FVA based on the application size together with a community approach to harvest PEMs to further reduce the risk of failure of legacy application migration.

3. A review of the proposed FVA with commercial TCO calculators (including from AWS, Azure and Dell) as well as TCO models from relevant research, resulting in an extensible unified FVA calculator that includes explicit as well as hidden one-off, recurring and dynamic cloud costs, and

4. Validation of the proposed FVA approach using two real world anonymised case studies.

The rest of the paper is organised as follows. Section 2 presents related work on the cloud TCO research and discusses their limitations and shortcomings. Section 3 presents an overview of the proposed approach with a specific focus on application sizing using the PEM and the subsequent FVA. Section 4 validates the framework's suitability by providing two real-world case studies. Section 5 concludes the paper by providing a summary of the completed work and identifying areas of future work.

\section{Related Work}

Current approaches for cloud migration tend to focus only on providing guidance on the technical recommendation or assume public cloud is the target cloud platform [20] [21]. If a calculator is provided, it is either for public cloud, e.g., AWS [10], Azure [11], or for on-premise private cloud, e.g., DELL [12] and Equinix [13], and they tend to be platform focused and not holistic. As cloud application solutions continue to fragment, with compute, storage, analytics and other services running in clouds from various providers with different pricing structures, cloud cost estimation becomes a non-trivial task [22]. Further complexity in cost estimation is driven by the two commercial models 
of PAYG and subscription-based models that can both have a committed spend, increasing year on year, with an enterprise agreement associated with them. The TCO models identified in [5] [22] fail to capture the majority of hidden costs such as the professional services costs required to specify the target cloud platform production/non-production environments, professional services required for setup of object storage, high performance storage, management and monitoring, backup, training, and platform software licensing. Furthermore, the new operating model requires new skills to support the platform, hence there is a requirement to factor in training costs to acquire new skills and toolsets to support this transition. From a commercial perspective, the TCO's scope is limited to public cloud and does not support application of bulk discounts for enterprise subscriptions. Our research not only addresses these shortcomings but also provides a more comprehensive approach to governing cloud migration via a framework that identifies the required application characteristics and tailors the FVA accordingly to mitigate the risk of budget overruns.

There is no doubt that elasticity in public cloud is an important feature to support scalability of resources, however it is only valuable if coupled with a reliable PAYG commercial model that can be used to predict the costs based on application characteristics [23]. Furthermore, even as service provider and research TCO models evolve, the approaches advocated in [5] [14] [22] are rigid, for example, being limited to the PAYG commercial model that risks distorting the comparison due to potential bulk discounts being available with enterprise discounts and reserved instances for yearly commitment available. This is further exacerbated when dealing with multiple cloud providers since one has to deal with network latency, management of disparate APIs, additional costs associated with keeping documentation up to date, and costs associated with data access within the public cloud provider and data transport outside of the public cloud provider or between regions of the public cloud provider.

Hidden costs are a concern for enterprises when they consider/plan to migrate their applications to Cloud Computing [3]. Identifying them in the planning phase will reduce the risk of cost overruns [24] and increase the probability of a sustainable cloud migration [24] [25]. Additional organisation costs that must be considered in cloud migration include learning, setup, additional network bandwidth to support data migration, and security costs to mitigate security risks [24]. In contrast to service provider calculators and research on TCO models for cloud that require individual resources to be nominated and quantities identified [10] [11] [12] [13], our approach proposes a PEM based on application sizing to help automate the forecast of cloud run costs in the planning phases of a project. The benefit of the parametric approach is to provide a technique to generalise the estimation process to a manageable level and enable confident, coordinated and efficient decision-making in the planning phase.

Current enterprise approaches tend to build a business case per application using existing service provider calculators together with information gleaned from cost optimisation tools as a guide based upon application profiles and usage [1] [26]. The limitation of this approach is that it does not consider the specific application characteristics in the context of projected costs. Cost optimisation tools assist with managing costs on a day-to-day basis, but clear linkage between business wants and needs, application characteristics and an FVA for cloud run costs is likely to assist in managing financial risks in the planning phase. To the best of our knowledge, our approach in this paper is the first to provide an integrated FVA methodology that is combined with a Cloud Decision Framework (CDF) to link platform choice with forecast costs. Importantly the FVA methodology addresses shortcomings in research to use applicable application characteristics and size to forecast cloud costs via the PEM. Using our approach, a cloud adopter can relatively quickly decide if a cloud migration project is viable or not. The governing of cloud migration and associated practices play an important role in the broader IT governance [6]. Our proposed framework provides decision support for the migration of legacy applications to hybrid cloud based on a ROM estimate of cloud run and migration costs. In doing so, it provides the key decision-makers the ability to better choose and prioritise their IT investments [27].

\section{Cloud Decision Framework with Financial Viability Assessment}

We propose a Cloud Decision Framework (CDF) to support the technical and commercial decision making of business users, business analysts and/or solution leads when choosing between public or private cloud for their application in the planning phase. As shown in Fig. 2, the decision-making process begins with the Wants and Needs Gathering step followed by Detailed Use Case Modelling. The application characteristics are ordered in the Criteria Classification step, either according to industry best practice or the enterprise's specific preferences. Next, a Technical Platform Recommendation (TPR) is generated based on the criteria classification using Rules Based Reasoning 


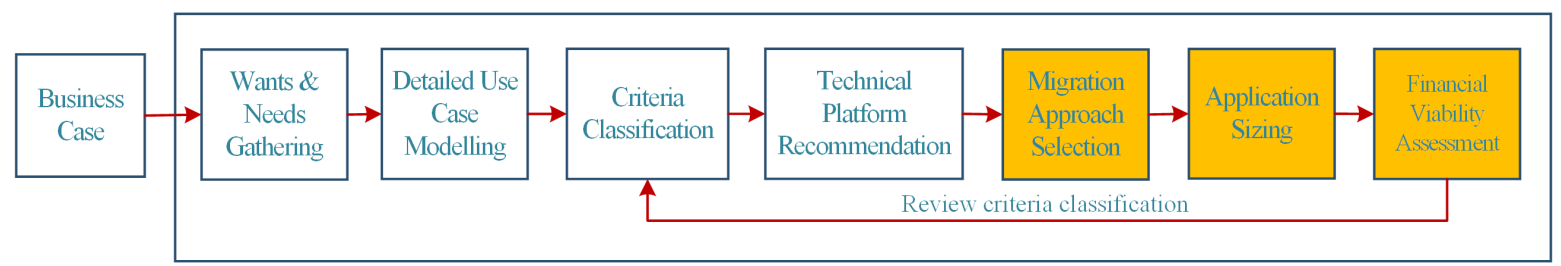

Figure 2. Cloud Decision Framework With Financial Viability Assessment.

(RBR), followed by the Financial Viability Assessment (FVA) of the recommended platform. By providing traceability of the cost items in the FVA calculator to the decision criteria, the framework enables users to iterate through the decision process, determining and eliminating the main cost drivers until a right balance is found between the desirable criteria and the available budget. If a right balance cannot be found, then users can either revert to the Wants and Needs Gathering step to reconsider the project criteria or abandon the project altogether.

We automate the FVA process via the Migration Approach Selection step (for determining migration to IaaS, PaaS and/or SaaS), followed by Application Sizing linked to a PEM that pre-populates the FVA calculator (shaded orange in Fig. 2). We do this by (a) adding support for a cloud adopter to select pre-configured application sizes following selection of a migration method, (b) elaboration of the FVA using a bottom-up approach to build the unified FVA calculator $^{1}$, (c) providing PEM support to automate the FVA calculator generation as much as possible and utilise community support for the PEM construct on an application basis, and (d) enabling the addition of $\mathrm{PaaS}$ and/or SaaS cost items as required for the purposes of comparison. It should be noted that the unified FVA methodology can be extended to also support other cloud providers. For each provider, the FVA methodology supports cost comparison across three application sizes - small, medium and large (refer to example in Table 1). Following this step, the hidden costs are listed in the calculator with an estimate for each application (or 't-shirt') size that is applicable.

\subsection{Parametric Estimation Model}

The PEM is typically represented in a three-point estimation format in which three figures are produced initially for every distribution, namely the best-case estimate, the most-likely estimate and the worst-case estimate to represent the outcomes of future events

\footnotetext{
${ }^{1}$ This research uses the cost criteria and formulas from publicly available calculators and the hidden costs of cloud as a starting point and then adds additional cost items to cater for PaaS and SaaS that are particular to the public cloud providers.
}

based on prior experience or best-guesses [28]. In the context of cloud migration, a PEM groups several "similar" applications and provides predefined sizes for them. The t-shirt sizing is influenced by the infrastructure requirements per application, noting multiple applications or components can be deployed to meet business outcomes with the infrastructure sized based on the number of parallel users to be supported and the data retention period required.

In addition to supporting IaaS, the FVA supports the service provider's PaaS and SaaS offerings. For example, the 'Database as a Service' PaaS offering (as a managed service) can be compared against installing a database system on a VM. For the PaaS and SaaS models, the PEM strongly depends on the cloud provider's offerings and, thus, is tightly coupled to the cloud provider. Moreover, if PaaS services such as Azure's Web Application Service are used, it is not a virtual machine (VM) that runs all the time (for which the cost can be calculated easily) but depends on the actual workload and the number of requests that are processed and therefore have to be forecast. As PaaS and SaaS services are tightly coupled with the service provider, each PEM is particular to an application and public cloud provider when these tiers are involved, hence custom items are introduced to cater for those resources or services. Each PEM will have an inherent dependency upon the migration approach whether that be 'lift and shift' to IaaS, 'transform' to PaaS or 're-architect' to cloud native. Hence, users are encouraged to adjust the default quantities for the application category.

It is challenging to find a suitable PEM for the breadth of applications that are considered for migration to the cloud. Therefore, we advocate a community-based approach to harvest PEM models as they are created by experts, improving the likelihood that PEMs can be found for the target application.

\subsection{Application Sizing}

Application sizing is the first step in assessing the financial viability of a cloud migration project. It can be achieved in two ways: a PEM-based automated way, 
Table 1. Automated Application Sizing.

\begin{tabular}{|l|l|l|l|}
\hline Application & Small & Medium & Large \\
\hline Application & Prod. \& 2 & Prod. \& 2 & Prod. \& 2 \\
& Non-Prod. & Non-Prod. & Non-Prod. \\
& env.: & env.: & env.: \\
& 20VMs, & 55VMs, & 80VMs, \\
& 10TB & 15TB & 30TB \\
\hline Application & Prod. \& 2 & Prod. \& 2 & Prod. \& 2 \\
2 & Non-Prod. & Non-Prod. & Non-Prod. \\
& env.: & env.: & env.: \\
& 50VMs, & 70VMs, & 90VMs, \\
& 41TB & 82TB & 123TB \\
\hline
\end{tabular}

or through a manual process. The technique used for application sizing is determined by the responses to the following two questions:

Q1: Are each of the (existing) application components supported in a virtual environment? The answer can be determined by using the following two-step process:

(a) Determine if any physical devices are supported as virtual appliances in the cloud platform from either the cloud providers or the Independent Software Vendor (ISV) of the application, and

(b) Identify the key hypervisor dependencies from the cloud provider.

Q2: Are each of the virtual components supported in the recommended cloud platform?

3.2.1. Automated Process: If the answer is 'yes' to both Q1 and Q2 then PEM can be used to assess the application size. The role of PEM in the decision-making process is to provide a means for classifying an application into a group or t-shirt size, which in turn provides a means of automating the FVA. Using a predefined model provides a balance between complexity and size for a specific application. Subject Matter Experts (SMEs) can create PEMs for different application architectures as required.

To illustrate the automated process for application sizing, let us consider two types of applications; Application 1 is a monolithic SAP ERP Central Component (ECC) deployment with the following VM profile - Production (7VMs, 2xLarge, 3xMedium, 2xSmall) and Non-production (2xMedium VMs, 2xSmall per SAP application), and Application 2 is a cloud-native deployment of Hadoop. Table 1 reflects the PEMs developed for both deployments in which an application has three predefined T-shirt sizes (Small, Medium or Large) based on the number of VMs required. The VM and storage estimates for these examples were obtained by averaging a sample of 15 enterprise implementations of SAP and Hadoop. Each
Table 2. Manual Application Sizing.

\begin{tabular}{|l|l|}
\hline $\begin{array}{l}\text { Number of Production } \\
\text { VMs }\end{array}$ & $45 \mathrm{VMs}+$ PaaS \\
\hline Storage Requirements & $16 \mathrm{~GB}$ \\
\hline $\begin{array}{l}\text { Unsupported } \\
\text { components }\end{array}$ & Bare Metal \\
\hline Redundant Instance & No \\
\hline $\begin{array}{l}\text { Lifecycle Requirements } \\
\text { Upgrade of operating system required } \\
\text { prior to managed service being } \\
\text { provided }\end{array}$ \\
\hline Monitoring & $\begin{array}{l}\text { Re-use } \\
\text { Current monitoring solution is } \\
\text { portable }\end{array}$ \\
\hline Commercial Model & $\begin{array}{l}\text { New licenses are required for the } \\
\text { upgrade }\end{array}$ \\
$\begin{array}{l}\text { Options: Reserved Instances (RIs) or } \\
\text { PAYG and PaaS } \\
\text { Choice: RIs and PaaS given the } \\
\text { application is strategic. }\end{array}$ \\
\hline
\end{tabular}

company's SAP and Hadoop SME was interviewed to obtain the number of VMs and storage required.

3.2.2. Manual Process: If the answer is 'no' to either Q1 or Q2, a manual process can be used to capture the quantities required for each cost item by following the steps described below:

- Capture the number of VMs required with CPU, memory and storage requirements by comparing the current deployment at peak periods, and requesting platform requirements from the ISV or development team (particularly when an upgrade is required as part of the migration).

- Capture any components not supported in a virtual environment after checking if there is no equivalent virtual appliance, the throughput required in the virtual appliance is not equivalent to the physical device, or the virtual appliance is not cost-effective.

- Capture whether a redundant second instance of the platform is required.

- Identify the lifecycle requirements of the platform by examining the ISV's support dates and dependencies.

- Determine if the current backup solution will be re-used by the target environment; alternatively, examine backup requirements and enter the quantities of the cost items (refer to Tables 3 \& 4) appropriately.

- Forecast consumption of relevant PaaS/SaaS services.

- Assess if the monitoring and managed service requirements continue or adjust the quantities as required.

- Leverage existing software licenses where possible.

To illustrate the manual process, we consider and elaborate on the scenario of a Life Insurance Advisor Quoting Application running on on-premise legacy infrastructure deployed on a virtual platform and bare metal. The adviser application has 8 components, 
Table 3. Explicit Cost Items considered in Provider Calculators and Research.

\begin{tabular}{|l|l|l|}
\hline \multicolumn{2}{|l|}{ Public Cloud Items } & Ingress \\
\hline Virtual Compute & Storage & Audit \\
\hline Egress & Backup & Custom Items \\
\hline DNS Services & Elasticity & \\
\hline Private Cloud Items & \multicolumn{2}{|l|}{} \\
\hline Compute & Storage & Data Centre Space \\
\hline Air Conditioning & Audit & Power \\
\hline Backup & Installation & Software Licensing \\
\hline Frame & Custom Items & \\
\hline
\end{tabular}

between 600 to 1000 (Source Lines of Code) each. The migration path for the advisor application is to re-factor using a combination of Microsoft Azure IaaS and PaaS - Azure Application Service Environment (ASE) for the web application tier, and Azure API Management for integration. The forecast for Microsoft PaaS ASE is 10 instances driven by the need to meet the Total Committed Memory (TCM) requirement for the overall application. The implication to end users of lowering the number of ASE instances is lower performance that negatively impacts end user experience. The answers to the initial questions Q1(a) and Q1(b) are 'yes' and 'no'. Table 2 shows the application sizing after working through the above guidelines.

\subsection{Financial Viability Assessment}

Following application sizing, the next step is to apply the FVA to generate the forecast costs. These costs include the cost items that are common to all cloud platform calculators (see Table 3) as well as the hidden costs identified after examining research, service provider calculators and costs incurred by enterprise (see Table 4). None of the research provided Models [14] [15] or service provider TCO calculators [10] [11] [12] [13] capture all of them.

\section{Validation}

We present two real-world examples to demonstrate the flexibility of our approach in assessing the financial viability of cloud migration projects. The total cost estimate is automatically calculated via the use of PEM, with an user having the option of modifying the required quantities for each cost item. Any hidden or custom $\mathrm{PaaS} / \mathrm{SaaS}$ costs are identified in Table 4

\subsection{Case Study 1}

The first case study relates to a Contact Centre running the Avaya platform on infrastructure that had reached end of maintenance support. Cloud Computing was pursued because the enterprise has a 'Cloud First' policy for all new upgrades or build of new applications.
Table 5 summarises the use case scenario and lists the criteria classification for the cloud decision.

- Technical Platform Recommendation: The application has an availability requirement higher than $99.95 \%$ with commercial ramifications for non-conformance. Hence, the CDF recommends private cloud, which is the default platform for applications with stringent QoS levels.

- Application Sizing: In this case, the recommended migration approach is 'lift and shift' to the target platform in 2 data centers. The T-shirt size is determined to be large, based on the Avaya PEM (cf. Table 6) where the number of VMs, storage and Avaya applications have been used as follows to automate the FVA: Avaya Call Manager (ACM) - 22 VMs, Avaya Interactive Voice Response (IVR) - 34 VMs and Avaya Workforce Optimisation (WO) - 40 VMs, 6 X Physical Servers for call recording.

- Generalised FVA Calculations: Based on the t-shirt size, the total cost for the two alternatives are presented in the individual worksheets in Tables 9 and 10. The cost items can be traced to the Cloud Decision Criteria in Table 6, as shown in the column titled 'Trace to Criteria', with the exception of those identified as Project Costs (PC). These costs are platform agnostic.

- Key Finding: The estimated cost for the alternatives of Managed Private Cloud and Managed Public Cloud over a 5-year period is $\$ 11.7 M$ and $\$ 17.1 M$ respectively. Based on the FVA outcome, the final decision is to recommend the migration of this application to private cloud because it is substantially less expensive than the alternative. Since the migration involved a well known and understood application, the forecast costs were equal to the actual costs once deployed to production.

\subsection{Case Study 2}

In this case study, the enterprise had a critical website running on dedicated infrastructure that reached full capacity during a marketing event due to a lack of horizontal scalability. This resulted in lost revenue due to outages experienced due to these capacity constraints. The peak requirements for this website were 3 million users and 20,000 concurrent sessions.

- Technical Platform Recommendation: Microsoft Azure was selected as the cloud service provider given the heritage of the website software (Microsoft .Net v4.5 and v4.6.2), lower risk of migration with Microsoft software components, the $80 \%$ discounts in-place for 3 years commitment for 
Table 4. Hidden Cost Items not considered in Provider Calculators.

\begin{tabular}{|c|c|c|c|}
\hline $\begin{array}{l}\text { Additional TCO } \\
\text { Model Cost Items }\end{array}$ & $\begin{array}{l}\text { Public } \\
\text { Cloud }\end{array}$ & $\begin{array}{l}\text { Private } \\
\text { Cloud }\end{array}$ & Review against research, service provider TCO \& Relevance \\
\hline \multicolumn{4}{|c|}{ Architecture \& Project Management Professional Services } \\
\hline $\begin{array}{l}\text { Solution Architecture } \\
\text { deliverable }\end{array}$ & $\checkmark$ & $\checkmark$ & $\begin{array}{l}\text { The professional services work required to capture the compute, storage, networking and } \\
\text { system software platform for an application with the management and monitoring solution. } \\
\text { This element is not applicable to a private cloud, since it is built in the factory based on } \\
\text { capacity requirements based on a reference architecture. }\end{array}$ \\
\hline $\begin{array}{l}\text { Project Management } \\
\text { Professional Services }\end{array}$ & $\checkmark$ & $\checkmark$ & $\begin{array}{l}\text { The Project Management professional services responsible for planning, budgeting and } \\
\text { resourcing for public cloud infrastructure detailed design. }\end{array}$ \\
\hline \multicolumn{4}{|c|}{ Network Connectivity \& Security } \\
\hline Security Services & $\checkmark$ & $\sqrt{ }$ & $\begin{array}{l}\text { Provides consideration for new identity, firewall, anti-virus or Denial of Service for public } \\
\text { or private cloud. }\end{array}$ \\
\hline DNS Services & $\checkmark$ & $\checkmark$ & $\begin{array}{l}\text { A scalable and highly available domain name that is part of a public or private cloud } \\
\text { platform service. }\end{array}$ \\
\hline $\begin{array}{l}\text { Connectivity to a } \\
\text { private network }\end{array}$ & $\checkmark$ & $x$ & $\begin{array}{l}\text { This resource captures the data networking between the public cloud network and the } \\
\text { enterprise's private network. }\end{array}$ \\
\hline \multicolumn{4}{|c|}{ Monitoring \& Management } \\
\hline Lifecycle Management & $x$ & $\checkmark$ & It is an IT Asset lifecycle to determine if the asset requires refresh or replacement. \\
\hline $\begin{array}{l}\text { Automation } \\
\text { Components }\end{array}$ & $\checkmark$ & $\checkmark$ & Establishment if automation components such as a Cloud Management Platform (CMP). \\
\hline $\begin{array}{l}\text { Governance } \\
\text { resources }\end{array}$ & $\checkmark$ & $x$ & A method of providing role-based access for the provisioning of cloud resources. \\
\hline \multicolumn{4}{|c|}{ Commercial Considerations } \\
\hline $\begin{array}{l}\text { Managed Private Cloud } \\
\text { resource overhead }\end{array}$ & $x$ & $\checkmark$ & $\begin{array}{l}\text { A headroom heuristic of } 30 \% \text { resource overhead caters for peak loads in a private cloud } \\
\text { deployment to minimise the risk of degraded performance. }\end{array}$ \\
\hline $\begin{array}{l}\text { High Availability } \\
\text { Disaster Recovery }\end{array}$ & $\checkmark$ & $\checkmark$ & $\begin{array}{l}\text { Public and Private Cloud Managed Service Providers will have dependencies upon } \\
\text { components to meet high availability requirements. }\end{array}$ \\
\hline Exchange Rate & $\checkmark$ & $x$ & $\begin{array}{l}\text { Both AWS and Microsoft Azure bill their services in United States dollars. A currency } \\
\text { conversion is critical to view in local currency as per our prototype. }\end{array}$ \\
\hline Software Licenses & $\checkmark$ & $\checkmark$ & $\begin{array}{l}\text { Microsoft tends to incentivise those with Microsoft technologies in being able to migrate } \\
\text { them to Azure. In this instance, it provides a competitive advantage over the AWS platform } \\
\text { and must be considered in forecasting cloud costs. }\end{array}$ \\
\hline Custom Items & $\checkmark$ & $x$ & $\begin{array}{l}\text { Any items considered important by the Cloud Adopter to be included that are above the } \\
\text { IaaS layer, such as PaaS and SaaS and specific to a public or private cloud. }\end{array}$ \\
\hline
\end{tabular}

Table 5. Case Study 1 Use Case Scenario.

\begin{tabular}{|c|c|c|}
\hline $\begin{array}{l}\text { Use } \\
\text { case } \\
\text { overview }\end{array}$ & \multicolumn{2}{|c|}{$\begin{array}{l}\text { A customer contact centre is effectively a } \\
\text { company's "store front". The end-to-end service } \\
\text { availability is required to be } 99.97 \% \text { per month } \\
\text { with critical transactions to be executed in less } \\
\text { than } 3 \text { seconds. }\end{array}$} \\
\hline \multirow{18}{*}{$\begin{array}{l}\text { Cloud } \\
\text { Decision } \\
\text { Criteria }\end{array}$} & Criteria & Classification \\
\hline & Availability (A) & Required \\
\hline & Business Service Availability (BSA) & Required \\
\hline & $\begin{array}{l}\text { Long running business process } \\
(\text { LRBP) }\end{array}$ & Required \\
\hline & Application Usage (AU) & Optional \\
\hline & Regulatory requirements (RR) & Required \\
\hline & Operating Costs (OC) & Optional \\
\hline & Performance $(\mathrm{P})$ & Optional \\
\hline & Application architecture (AA) & Required \\
\hline & Application constraints (AC) & Required \\
\hline & Security (S) & Required \\
\hline & Data Security Classification (DSC) & Optional \\
\hline & $\begin{array}{l}\text { Network Global Load Balancing } \\
\text { (NGLP) }\end{array}$ & Optional \\
\hline & $\begin{array}{l}\text { Connectivity to private } \\
\text { network or internet VPN (C) }\end{array}$ & Optional \\
\hline & Hypervisor (HV) & Required \\
\hline & Enterprise Control (EC) & Required \\
\hline & Data Classification (DC) & Required \\
\hline & Technology Standardisation (TS) & Required \\
\hline
\end{tabular}

compute resources, and to leverage the elasticity of the platform using the PaaS features. Of the three migration options available (cf. Table 7), the 'refactor' option was selected to enhance the application, and a custom entry was added in the FVA calculator for Azure PaaS Web Application Service, Managed $S Q L^{*}$ Server DB and Managed Cosmos Db
Table 6. Case Study 1 PEM (Production, $2 \times$ Non-production environments)

\begin{tabular}{|l|l|l|l|}
\hline & Small & Medium & Large \\
\hline $\begin{array}{l}\text { Avaya } \\
\begin{array}{l}\text { Contact } \\
\text { Centre }\end{array}\end{array}$ & 1. Avaya CM & 1.Avaya CM & 1.Avaya CM \\
& & 2. Avaya IVR & $\begin{array}{l}\text { 2.Avaya IVR } \\
\text { 3.Avaya WO }\end{array}$ \\
\cline { 2 - 4 } & & & 64VMs, 10TB \\
& Storage & 162VMs, & 15TM Storage \\
40TB Storage \\
\hline
\end{tabular}

Table 7. Case Study 2 - Migration Options.

\begin{tabular}{|c|c|c|}
\hline Lift \& Shift & Refactor & Rebuild/Re-architect \\
\hline usive) & & ntrusive) \\
\hline $\begin{array}{l}\text { Migrate Net } \\
\text { application to } \\
\text { Azure IaaS as-is }\end{array}$ & $\begin{array}{lr}\text { Net application } \\
\text { deployed r to } \\
\text { Microsoft App } \\
\text { Service using } \\
\text { Containers }\end{array}$ & $\begin{array}{lr}\text { Migrate } & \text {.Net } \\
\text { application } & \\
\text { transformed } & \text { using } \\
\text { Serverless } & \text { and } \\
\text { Containers } & \end{array}$ \\
\hline
\end{tabular}

Table 8. Case Study 2 PEM.

\begin{tabular}{|l|l|l|l|}
\hline $\begin{array}{l}\text { Application } \\
\text { Complexity }\end{array}$ & Small & Medium & Large \\
\hline Microsoft & .Net custom & .Net custom & .Net custom \\
.Net & developed & developed & developed \\
Application & application & application & application \\
Profile & with & with & with \\
& Microsoft & Microsoft & Microsoft \\
& SQL*Server & SQL*Server & SQL*Server \\
& Database & Database & Database \\
\cline { 2 - 4 } & 10VMs, 1TB & 25VMs, 2TB & 50VMs, \\
& Storage & storage & 2TB Storage \\
\hline
\end{tabular}

(cf. Table 11).

- Application Sizing: The existing infrastructure had 30 VMs, whereas following migration, the application 
Table 9. Private Cloud Calculator.

\begin{tabular}{|c|c|c|c|c|}
\hline & Trace to Criteria & Upfront & Monthly Unit Cost & Qty \\
\hline \multicolumn{5}{|l|}{ Architecture \& PM } \\
\hline Solution Architecture & $\mathrm{PC}$ & $\$ 0.00$ & $\$ 0.00$ & 0 \\
\hline Project Management & $\mathrm{PC}$ & $\$ 0.00$ & $\$ 0.00$ & 0 \\
\hline Connectivity & & $\$ 0.00$ & $\$ 0.00$ & 0 \\
\hline Design \& Impl. (PROD) & $\mathrm{PC}$ & $\$ 647,500.00$ & $\$ 0.00$ & 1 \\
\hline Design \& Impl. (DR) & $\mathrm{PC}$ & $\$ 647,500.00$ & $\$ 0.00$ & 1 \\
\hline \multicolumn{5}{|l|}{ Compute \& Storage (PROD) } \\
\hline Vblock 350 Frame & A, BSA, LRBP & $\$ 0.00$ & $\$ 20,800.00$ & 1 \\
\hline Virtual Blades & $\begin{array}{l}\text { A, BSA, LRBP, HV, AA, } \\
\text { AU, AC }\end{array}$ & $\$ 0.00$ & $\$ 2,450.00$ & 8 \\
\hline Physical Blades & A, BSA, LRBP & $\$ 0.00$ & $\$ 1,150.00$ & 4 \\
\hline Storage & A, BSA, LRBP & $\$ 0.00$ & $\$ 7,450.00$ & 1 \\
\hline \multicolumn{5}{|l|}{ Compute \& Storage (DR) } \\
\hline Vblock 350 Frame & A, BSA, LRBP & $\$ 0.00$ & $\$ 29,450.00$ & 1 \\
\hline Virtual Blades & $\mathrm{A}, \mathrm{BSA}, \mathrm{LRBP}, \mathrm{H}, \mathrm{AR}$ & $\$ 0.00$ & $\$ 2,450.00$ & 8 \\
\hline Physical Blades & A, BSA, LRBP & $\$ 0.00$ & $\$ 1,150.00$ & 4 \\
\hline Storage & A, BSA, LRBP & $\$ 0.00$ & $\$ 7,450.00$ & 1 \\
\hline Ingress \& Egress Fees & $\mathrm{C}$ & $\$ 0.00$ & $\$ 0.00$ & 0 \\
\hline Monitoring \& Management & $\mathrm{PC}$ & $\$ 0.00$ & $\$ 25,000.00$ & 1 \\
\hline \multicolumn{5}{|l|}{ Data Centre } \\
\hline Power & $\mathrm{OC}, \mathrm{RR}$ & $\$ 0.00$ & $\$ 3000.00$ & 7 \\
\hline Cooling & $\mathrm{OC}, \mathrm{RR}$ & $\$ 0.00$ & $\$ 3000.00$ & 7 \\
\hline Floor Space & $\mathrm{OC}, \mathrm{RR}$ & $\$ 0.00$ & $\$ 3000.00$ & 7 \\
\hline \multicolumn{5}{|l|}{ Lifecycle Management } \\
\hline Platform engineering & $\mathrm{PC}$ & $\$ 1,200.00$ & $\$ 0.00$ & 0.5 \\
\hline Incident management \& remediation & $\mathrm{PC}$ & $\$ 0.00$ & $\$ 0.00$ & 0 \\
\hline Platform migration & $\mathrm{PC}$ & $\$ 1,200.00$ & $\$ 0.00$ & 20 \\
\hline Pre-deployment validation lab & $\mathrm{PC}$ & $\$ 0.00$ & $\$ 0.00$ & 0 \\
\hline Backup & $\mathrm{PC}$ & $\$ 0.00$ & $\$ 128.57$ & 44 \\
\hline Contract Administration & $\mathrm{PC}$ & $\$ 0.00$ & $\$ 500.00$ & 1 \\
\hline Audit & $\mathrm{PC}$ & $\$ 0.00$ & $\$ 300.00$ & 1 \\
\hline Yearly Price & $\$ 2,350,086.00$ & 5 Year Total & $\$ 11,750,428.00$ & \\
\hline
\end{tabular}

Table 10. Public Cloud (AWS) Calculator.

\begin{tabular}{|c|c|c|c|c|}
\hline & Trace to Criteria & Upfront & Monthly Unit Cost & Qty \\
\hline \multicolumn{5}{|l|}{ Architecture \& PM } \\
\hline Project Management & $\mathrm{PC}$ & $\$ 0.00$ & $\$ 1,200.00$ & 20 \\
\hline Design \& Impl. (PROD) & $\mathrm{PC}$ & $\$ 50,000.00$ & $\$ 0.00$ & 1 \\
\hline \multicolumn{5}{|l|}{ Compute \& Storage (PROD) } \\
\hline Infrastructure & $\begin{array}{l}\text { A, BSA, LRBP, HV, AA, AU, } \\
\text { AC }\end{array}$ & $\$ 0.00$ & $\$ 1,000.00$ & 1 \\
\hline Storage & A, BSA, LRBP & $\$ 0.00$ & $\$ 100.00$ & 44 \\
\hline \multicolumn{5}{|l|}{ Compute \& Storage (DR) } \\
\hline Infrastructure & $\begin{array}{l}\text { A, BSA, LRBP, HV, AA, AU, } \\
\text { AC }\end{array}$ & $\$ 0.00$ & $\$ 1,000.00$ & 1 \\
\hline Virtual Compute & A, BSA, LRBP & $\$ 0.00$ & $\$ 486.06$ & 280 \\
\hline Storage & A, BSA, LRBP & $\$ 0.00$ & $\$ 100.00$ & 44 \\
\hline Cooling & NA & $\$ 0.00$ & $\$ 0.00$ & 0 \\
\hline Floor Space & NA & $\$ 0.00$ & $\$ 0.00$ & 0 \\
\hline \multicolumn{5}{|l|}{ Lifecycle Management } \\
\hline Platform engineering & $\mathrm{PC}$ & $\$ 1,200.00$ & $\$ 0.00$ & 1 \\
\hline Incident management \& remediation & $\mathrm{PC}$ & $\$ 50,000.00$ & $\$ 0.00$ & 1 \\
\hline Platform migration & $\mathrm{PC}$ & $\$ 1,200.00$ & $\$ 0.00$ & 40 \\
\hline Pre-deployment validation lab & $\mathrm{PC}$ & $\$ 0.00$ & $\$ 0.00$ & 0 \\
\hline Backup & $\mathrm{PC}$ & $\$ 0.00$ & $\$ 223.57$ & 44 \\
\hline Contract Administration & $\mathrm{PC}$ & $\$ 0.00$ & $\$ 500.00$ & 1 \\
\hline Audit & $\mathrm{PC}$ & $\$ 6000.00$ & $\$ 600.00$ & 1 \\
\hline Yearly Price & $\$ 3,416,199.00$ & 5 Year Total Price & $\$ 17,080,997.00$ & \\
\hline
\end{tabular}

consisted of $25 \mathrm{VMs}$ at the IaaS layer (as a mix of small, medium and large VMs), and PaaS for the web tier using Web Application Service. The 25 VMs map to the 'Medium' t-shirt size in the .Net PEM (see Table. 8). The .Net PEM has been created through examining 30 application migration projects which 
Table 11. Public Cloud (Microsoft Azure) laaS \& PaaS Calculator.

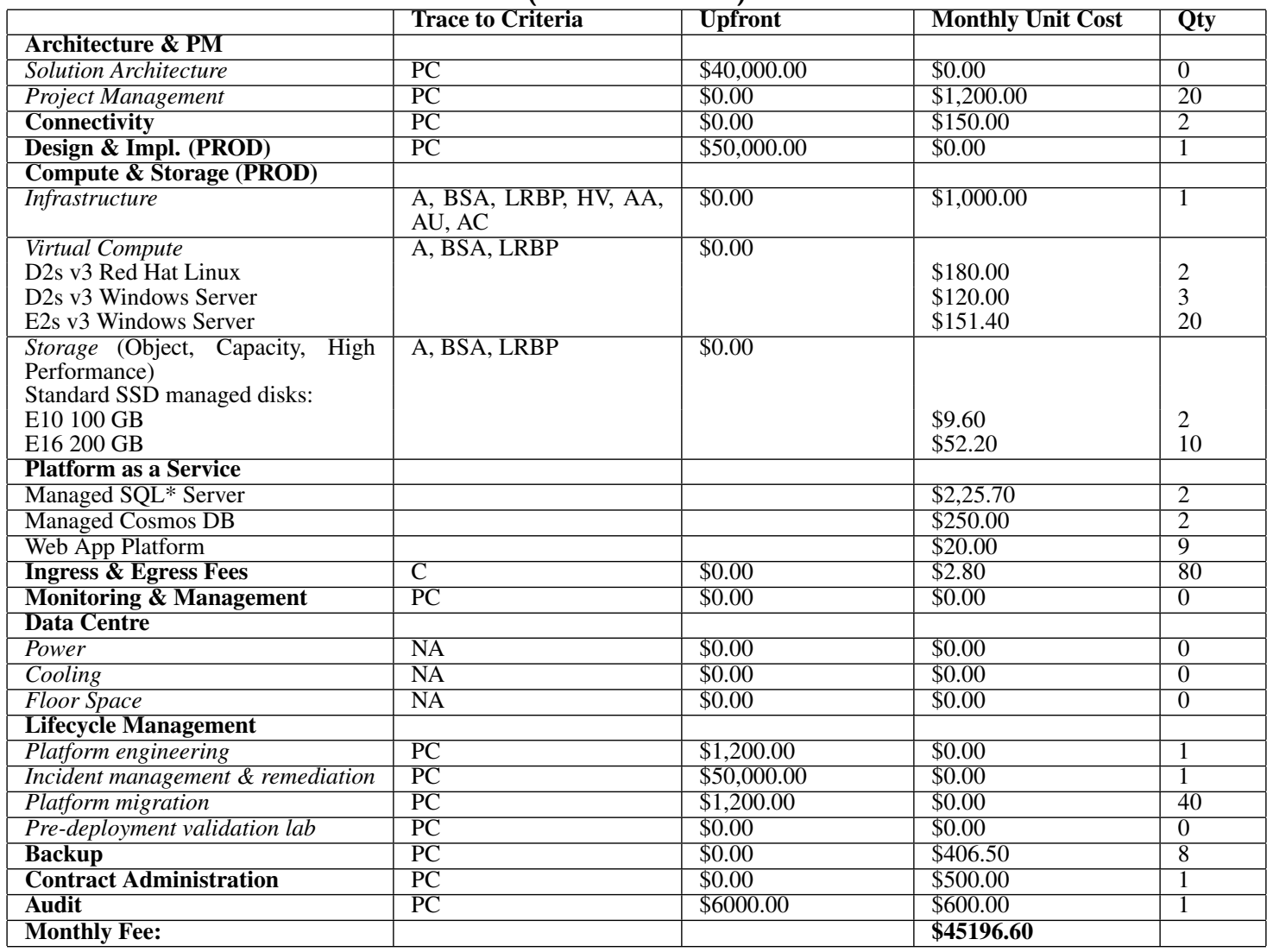

have been observed as a domain SME.

- Key Finding: The target platform FVA estimate of $\$ 45,196 /$ month (see Table 11) is lower than the as-is platform cost of $\$ 65,000 /$ month. Taking the lost revenue into consideration, the cloud platform is even more appealing. Once deployed to Microsoft Azure the actual cost was $\$ 51,590 /$ month due to a higher quality of service being required in the Web Application Service PaaS function, to cater for equivalent end user experience to the legacy platform.

The results for Case Study 1 and Case Study 2 are in-line with industry-accepted best-practice recommendations by Gartner [5], which recommends balancing the need for expediency and precision to achieve the most appropriate level of accuracy for cloud migration without expending too much effort. The forecast ROM cloud run costs for both case studies (\$11.7 million/year and $\$ 45,196 /$ month), are within $15 \%$ of the actual costs ( $\$ 11.7$ million/year and $\$ 51,590 /$ month), demonstrating that the framework provides a result within an acceptable order of magnitude [5] [29].

\section{Conclusion}

Enterprises are challenged in determining the suitability of an application for on-premise private cloud or public cloud, which could be managed by either the enterprise itself or the cloud provider. Comparing these options is problematic, not only due to the number of application characteristics requiring consideration, but also because each of them have characteristics that impact a Cloud TCO model. In this paper, we presented a CDF incorporating FVA to identify fit for purpose cloud infrastructure for applications being deployed to the cloud. In doing so, we addressed a gap in current cloud economics research by identifying key hidden costs that are incurred by an enterprise and should be considered in the FVA. The proposed approach uses application sizing via PEMs to automate the FVA process. We also proposed a community-based approach for harvesting application PEMs to reduce cloud migration risks.Application of the proposed CDF to two real-world anonymised case studies indicates that it is more likely to reduce budget overruns in cloud migration projects. The next focus of our work is to perform extensive testing and validation using our 
prototype.

\section{References}

[1] Flexera, "Flexera 2020 State of the Cloud Report." https://info.flexera.com/SLO-CM-REPORT-State-ofthe-Cloud-2020, 2020.

[2] D. Brue, "Public cloud costs push CBA to Openstack." www.itnews.com.au/news/public $=$ cloud-costs-push -cba-to-openstack-464239, 2017. [Online: accessed September 3, 2018].

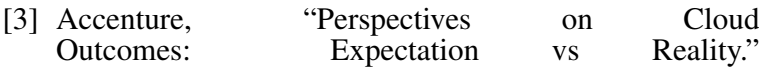
https://www.accenture.com/be-en/insights/cloud/ cloud-outcomes-perspective, 2020. [Online: accessed August 15, 2020].

[4] P. Rosati, G. Fox, D. Kenny, and T. Lynn, "Quantifying the financial value of cloud investments: a systematic literature review," International Conference on Cloud Computing Technology and Science, pp. 194-201, 2017.

[5] M. Meinardi, "How to Develop a Business Case for the Adoption of Public Cloud Iaas," Gartner Research, 2018.

[6] S. De Haes and W. Van Grembergen, "IT Governance Structures, Processes and Relational Mechanisms: Achieving IT/Business Alignment in a Major Belgian Financial Group," pp. 237b-237b, 2005.

[7] J. Rakowski, "Critical KPI's for Successful Cloud Migration.” White Paper, 2017.

[8] S. Balaji and S. Murugaiyan, "Waterfall vs V-Model vs Agile: A Comparative Study on SDLC," International Journal of Information Technology and Business Management, pp. 26-30, 2012.

[9] Project Management Institute, A Guide to the Project Management Body of Knowledge. Project Management Institute, 2017.

[10] Amazon Web Services, "Simple Monthly Calculator." https://calculator.s3.amazonaws.com/index.html, 2020. [Online: accessed on December 20, 2019].

[11] Azure, "Azure pricing calculator." https://azure.microsoft.com/pricing/calculator, 2019. [Online: last accessed December 12, 2019].

[12] DELL, "The ROI of Private Cloud." https://www.emc.com/collateral/white-papers/h15537 -the-roi-of-private-cloud-wp.pdf, $2017 . \quad$ [Online: accessed October 10, 2019]].

[13] Equinix, "TCO Calculator." https://www.equinix.com.au, 2019. [Online: accessed October 10, 2019].

[14] R. Makhlouf, "Cloudy transaction costs: a dive into cloud computing economics," Journal of Cloud Computing, p. Article 1, 2020.

[15] A. Soni and M. Hasan, "Pricing schemes in cloud computing: a review," International Journal of Advanced Computer Research, pp. 60-70, 2017.

[16] K. Ramchand, M. Baruwal Chhetri, and R. Kowalczyk, "Towards a comprehensive cloud decision framework with financial viability assessment," In Proceedings of the 22nd Pacific Asia Conference on Information Systems (PACIS 2018), p. p. 2744, 2018.

[17] K. Ramchand, M. Baruwal Chhetri, and R. Kowalczyk, "Towards a flexible cloud architectural decision framework for diverse application architectures," In The 28th Australasian Conference on Information Systems, (ACIS2017), WestPoint Tasmania, Hobart, Tasmania, 2017.

[18] K. Ramchand, M. Baruwal Chhetri, and R. Kowalczyk, "Towards a cloud architectural decision framework using case-based reasoning and rule-based reasoning," In The 29th Australasian Conference on Information Systems, (ACIS2018), University of Technology Sydney (UTS), Sydney Australia, 3-5 December, 2018.

[19] D. Ackerman, S. Graham, C.and Nag, and E. Anderson, "Market Share Analysis: IaaS and IUS," Gartner Research, 2019.

[20] M. Kavis, Architecting the Cloud: Design Decisions for Cloud Computing Service Models (SaaS, PaaS, and IaaS). John Wiley Sons, 2014.

[21] C. Pahl and H. Xiong, "Migration to paas clouds - migration process and architectural concerns," 7th International Symposium on the Maintenance and Evolution of Service-Oriented and Cloud-Based Systems, 2013.

[22] P. Maresova, V. Sobeslav, and O. Krejcar, "Cost-benefit analysis - evaluation model of cloud computing deployment for use in companies," Journal of Applied Economics, pp. 521-533, 2017.

[23] S. Safavi and U. Maqbool, "Effort estimation model for each phase of software development life cycle," Handbook of Research on E-Services in the Public Sector: E-Government Strategies and Advancements, pp. 270-277, 2017.

[24] A. Alhammadi, "A Knowledge Management Based Cloud Computing Adoption Decision Making Framework," PhD Thesis, 2016.

[25] A. Rahman, A. Islam, S. Kalloniatis, and C. Gritzalis, "A risk management approach for a sustainable cloud migration," Journal of Risk and Financial Management, v10, Issue 4, 2017.

[26] A. Menychtas, C. Santzaridou, G. Kousiouris, T. Varvarigou, et al., "Artist methodology and framework: a novel approach for the migration of legacy software on the cloud," pp. 424-431, 2013.

[27] P. Weill and J. Ross, IT Governance: How Top Performers Manage IT Decision Rights for Superior Results. Harvard Business School Press, 2004.

[28] D. Billows, Essentials of Project Management. The Hampton Group Inc, 2004.

[29] M. Meinardi and T. Clayton, "How to Manage and Optimize Costs of Public Cloud IaaS and PaaS," Gartner Research, 2020. 